\title{
Emu oil-based lotion effects on neonatal skin barrier during transition from intrauterine to extrauterine life
}

This article was published in the following Dove Press journal:

Clinical, Cosmetic and Investigational Dermatology

10 August 2017

Number of times this article has been viewed

\author{
Vincenzo Zanardo' \\ David Giarrizzo ${ }^{2}$ \\ Francesca Volpe \\ Lara Giliberti' \\ Gianluca Straface \\ 'Division of Perinatal Medicine, \\ Policlinico Abano Terme, Abano Terme, \\ ${ }^{2}$ CALANTHA Physiology of Lactation \\ Laboratory, Padua, Italy
}

\begin{abstract}
Both appropriate hydration and skin surface $\mathrm{pH}$ are fundamental in preventing baby skin barrier damage during transition from intrauterine to extrauterine life. However, effects of topical moisturizers on neonatal stratum corneum temperature, $\mathrm{pH}$, hydration, and elasticity have not been scientifically evaluated in vivo. We checked 31 full-term breastfeeding neonates by non-invasive bioengineering method, which is able to evaluate the basal skin barrier (left heel), and assessed at $6 \pm 1$ hours after birth, and at 1 and 24 hours after emu oil-based topical treatment. The basal skin barrier of right heel (no oil exposure) of each newborn was considered as control. We found that a single application of an emu oil-based lotion was effective in improving heel stratum corneum hydration, which increases both skin $\mathrm{pH}$ and elasticity without any effect on temperature. Further studies are needed to confirm long-term beneficial effects of this treatment in a very sensitive patient population.
\end{abstract}

Keywords: skin barrier, neonate, emu oil-based lotion, topical treatment

\section{Introduction}

The human newborn infant exhibits a fully formed and competent epidermal barrier at birth, despite its development in an aqueous environment. Biomedical evaluation indicates that transepidermal water loss values in term infants are equal to or lower than those of adults, demonstrating an excellent epidermal barrier integrity at birth. ${ }^{1}$ Although full-term healthy neonates have an anatomically well-developed skin, the postnatal epidermal barrier is functionally different from the fetal one as it is more prone to resist to environmental stress of extrauterine life, such as dryness and irritation..$^{2-4}$ Appropriate skin care is thus of relevant importance in neonates to maintain the physiological adaptation of skin barrier. ${ }^{5}$ Although several studies have shown that emollient therapy improves barrier function in premature neonates, ${ }^{6-8}$ very few studies have investigated the routine use of cleansers and emollients on skin of healthy, full-term neonates. ${ }^{7}$ Skin care regimens vary; they are usually based on tradition in many countries, and the influence of commercially available topical baby products on skin barrier function has not been scientifically investigated during the neonatal period. Some regimens may damage, and others may maintain barrier function. ${ }^{6-8}$ As a consequence, there is the need to characterize neonatal barrier function early in the extrauterine life. Appropriate baby skin hygiene and hydration are key factors in maintaining the barrier function and overall health. ${ }^{9,10}$ Proper cleaning keeps skin free of irritants, dirt, and germs. This is especially true in neonates, who have a higher surface area-to-volume ratio than adults and are more susceptible to infection. ${ }^{11}$
Correspondence: Vincenzo Zanardo Division of Perinatal Medicine, Policlinico Abano Terme, Piazza Colombo I, 3503 Abano Terme, Italy

Tel/fax +3949720027

Email vincenzo.zanardo@libero.it 
Since the postnatal maturation of the skin barrier in newborns underlines the necessity to use cleaners from the postpartum period until the end of the first years of life, our study was aimed to characterize neonatal barrier function. We used an emu oil baby moisturizer to test the effects on stratum corneum hydration, skin $\mathrm{pH}$, and elasticity in the neonate's heel region during the critical phase of transition and adaptation from intrauterine to extrauterine life. We hypothesized that the daily bathing with or without commercially available baby wash gel and additional emu oil baby moisturizer would not harm the natural adaptation of skin barrier in healthy newborns. Several studies have shown that emu oil, a commercial product available in many countries, seems to have various beneficial activities such as local enhancement of skin permeation and moisturizing and cosmetic properties. For these reasons, it has been recommended for the treatment of various ailments for decades. ${ }^{12,13}$ Topical use of emu oil, which contains high quantities of polyunsaturated fatty acids and antioxidants, ${ }^{14}$ seems to have potent anti-inflammatory effects. ${ }^{14,15}$ However, the way it interacts is not entirely clear, and up to now, it has not been extensively studied in neonates.

Therefore, the final aim of this study was to investigate the neonatal tolerance and dermal effects of a new emu oilbased lotion formulated especially for healthy infants. The study's primary goal was to test the related physiological characteristics of baby heel skin, hydration, $\mathrm{pH}$, temperature, and elasticity.

\section{Materials and methods}

This prospective, open-label, controlled clinical trial was carried out from March 1, 2015, to April 30, 2015, at the Policlinico Abano Terme (Abano Terme, Grandate, Italy) institution certified by the National Observatory for Women's Health (ONDA), an association dedicated to raising awareness on the importance of preventing and caring for illnesses afflicting women. The study was performed in accordance with the principles of the Declaration of Helsinki. All eligible mothers gave informed consent, and an institutional review board (Policlinico Abano Terme) approved the protocol. The trial was aimed at evaluating the tolerance and properties of an emu oil-based lotion in healthy, at term, breastfed neonates at the end of the first hours of extrauterine life.

The commercial name of the lotion used is Olio Emudermico $^{\circledR}$ (ELLEDIFRI Laboratories, Grandate, Italy) ${ }^{16}$ targeted in particular for newborns, infants, and young children. This formulation contains $20 \%$ of pure emu oil ${ }^{16}$ with high levels of omega- 3 and omega- 6 having a variety of beneficial effects such as anti-inflammatory and antioxidant properties. ${ }^{13,14}$ The product is free of fragrance and certified by the manufacturer as safe and appropriate for babies when the use was direct. The $\mathrm{pH}$ of lotion is neutral $(\mathrm{pH}$ 6.5) and authorized by the manufacturer for dermal and ocular safety, including clinical assessment for dermal irritation, dermal sensitization potential, and ocular irritancy and sting. ${ }^{19}$

The study's eligibility criteria included being a healthy singleton infant, who completed at least 37 weeks of gestation, without complications at birth and delivered between $6 \mathrm{am}$ to $6 \mathrm{pm}$ on a business day. The exclusion criteria was infants suffering from any known abnormal skin at baseline. After checking for inclusion and exclusion criteria, 31 mother-infant pairs were enrolled.

Women whose neonates fulfilled the eligibility criteria were counseled in the maternity ward by a researcher and invited to take part in the trial. Those who agreed and signed consent forms were enrolled and received instructions 6 hours after delivery (washout period). After the enrolment into the study, baseline measurements were performed.

The study was carried out within the context of the hospital's ordinary care protocol. In accordance with the hospital's standard practice, following an uneventful delivery, infants are placed on the mother's chest for $\sim 15$ minutes during which time the midwife assists with the first suckling episode. Infants are then dried, they receive umbilical care, and they are weighed before their first warm water bath. During the subsequent 2 days in the ward, newborns room-in with their mothers, who are encouraged to feed on demand (with no more than 3-hour interfeeding intervals). The infants received complementary formula milk if the midwives judged breast milk intake is insufficient.

Eligible mothers were instructed to apply the emu oilbased lotion to the neonates only on the left heel but not on the right one at $6 \pm 1$ hour after delivery. Each neonate formed thus both part of the study: case and control population. At the baseline visit, a pediatrician assessed infant skin condition before lotion application (baseline measurement) for erythema, dryness, rash, or overall skin condition at baseline in presence of the mother. The same pediatrician subsequently assessed the infant's heels at 1 and 24 hours after lotion application and tested skin with a Corneometer (Soft Plus 5.5; Callegari SPA, Parma, Italy). ${ }^{17}$ The test was performed in a fixed point (the heel of both feet) and included four parameters: temperature, $\mathrm{pH}$, hydration, and elasticity measurements. ${ }^{17}$

\section{Statistical analysis}

A descriptive analysis was used to construct a qualitative evaluation of clinical data. Continuous data were expressed 
as mean and standard deviation (SD) and compared using the Student's $t$-test. A $p$-value $<0.05$ was considered significant. Statistical analysis was performed using R 2.12 software. (Foundation for Statistical Computing, Vienna, Austria)

\section{Results}

A total of 31 consecutive infants were included in the study analysis. One infant, discharged with the mother at 12 hours after delivery, was not included. In addition, two infants were not included in the study as they were treated for a skin care regimen unrelated to the emu oil application. Half of the neonates were female $(56 \%)$, most of them were born by vaginal delivery (75\%), and the majority of mothers (98\%) breastfed their baby. The mean body weight loss after 24 hours was $7 \%$.

Skin care regimens did not harm physiological neonatal skin barrier adaptation. The condition of the skin based on the clinical conventional parameters assessed by pediatricians and by participant mothers was unaffected through the test.

The dermatological characteristics of the infants' heels who formed both the case and the control population of the emu oil-based lotion study are described in Table 1.

After 1 hour, emu oil-moisturized heels showed significantly higher $\mathrm{pH}$, hydration, and elasticity levels. Skin temperature was instead unaffected.

After 24 hours, despite neonatal body weight decrease of $\sim 7 \%$, emu oil-moisturized heels showed persistently significantly higher $\mathrm{pH}$, hydration, and elasticity. Again, skin temperature was unaffected.

\section{Discussion}

In this study, biomedical skin barrier evaluation in heel of healthy, full-term neonates revealed that a single application of an emu oil-based moisturizer was effective in improving stratum corneum hydration, $\mathrm{pH}$, and elasticity after 1 and even more after 24 hours of application. These data were confirmed by subjective evaluation of mothers.

The human heel skin is easily accessible to the observer. It is a thin, flexible, mechanically tough membrane, which is useful to test the protection against environmental aggressions and the loss of body water corresponding to physiological postnatal body weight decrease. Hydration and $\mathrm{pH}$, variables selected for quantitative comparison, revealed benefit of treated skin heel after 24 hours of the study without application of other baby lotions.

Infant moisturizing is a routine, culturally based practice involving exposure of the entire skin surface to natural or synthetic moisturizing agents in North-Eastern Italy. It is crucial to encourage mothers to prevent and treat the dry and desquamate skin of the neonate. However, the way that emu oil lotion interacts biophysically is not entirely clear. ${ }^{18-20}$

It is well known that the skin barrier function, which represents the first protection from water loss from the body, depends on several factors, including the level of hydration, the cellular properties of the corneocytes, the association and turnover of these cells in the stratum corneum, the amount and composition of intercellular lipids, and skin surface $\mathrm{pH} .{ }^{21}$ Moreover, the water content of stratum corneum and the skin surface lipids form a balance that is very important for skin function and integrity. ${ }^{22}$

Although scientific studies have not been designed to investigate emu oil's moisturizing effect on neonatal skin hydration, we found that there was an improvement in the stratum corneum hydration of the breast areolae 24 hours after emu oil-based cream application in different breastfeeding settings and that it was more pronounced in the puerperae presenting with basal hydration at the lower quartiles. ${ }^{23}$ The results posed questions about the cumulative effects of repeated wet-dry-wet exposures, such as those occurring

Table I Dermatological characteristics of the infants' heels who formed both part of the emu oil-based lotion study treatment and control population

\begin{tabular}{|c|c|c|c|c|c|}
\hline \multirow{3}{*}{$\begin{array}{l}\text { Neonates }(n=3 I) \\
\text { Heel's skin }\end{array}$} & \multirow{3}{*}{$\begin{array}{l}\text { Pretreatment } \\
\text { Baseline }\end{array}$} & \multicolumn{4}{|c|}{ Posttreatment } \\
\hline & & \multicolumn{2}{|l|}{ I hour } & \multicolumn{2}{|l|}{24 hours } \\
\hline & & Treated & Control & Treated & Control \\
\hline Hydration, cu & $35.0( \pm 15.4)$ & $42.9( \pm 15.6)^{*}$ & $33.7( \pm \mid 4.5)^{\#}$ & $46.8( \pm 16.7)^{* *}$ & $35.3( \pm 12.9)^{\# \#}$ \\
\hline $\mathrm{pH}$, units & $4.5( \pm 0.2)$ & $4.7( \pm 0.2)^{\circ}$ & $4.7( \pm 0.4)$ & $4.9( \pm 0.4)^{\circ \circ}$ & $4.7( \pm 0.3)$ \\
\hline Elasticity, cu & $45.4( \pm 2.1)$ & $45.8( \pm 2.2)$ & $44.3( \pm 2.1)^{@}$ & $46.6( \pm 1.6)^{\wedge}$ & $45.6( \pm I .4)^{@ @ ~}$ \\
\hline Temperature, ${ }^{\circ} \mathrm{C}$ & $29.6( \pm 0.9)$ & $30.0( \pm 1.0)$ & $30.0( \pm 1.0)$ & $30.1( \pm 0.7)$ & $30.0( \pm 0.8)$ \\
\hline \multirow[t]{2}{*}{$p$} & & \multicolumn{2}{|c|}{ Treated vs. control: $0.02^{\#}$} & \multicolumn{2}{|c|}{ Treated vs. control: $0.005^{\#}$} \\
\hline & & \multicolumn{2}{|c|}{ Treated vs. control: 0.01@ } & \multicolumn{2}{|c|}{ Treated vs. control: 0.01@@ } \\
\hline
\end{tabular}

Notes: $p$, statistical significance $<0.05$. *Baseline vs I hour: $\mathrm{p}=0.05 ; * *$ Baseline vs 24 hours: $\mathrm{p}=0.0 \mathrm{I} ;{ }^{\circ} \mathrm{pH}$, units: baseline vs I and 24 hours; ${ }^{\circ} \mathrm{pH}$, units: baseline vs 24 hours; ^elasticity, conventional units: baseline vs 24 hours; "hydration, conventional units: treated vs. control at I hour; "\#ydration, conventional units: treated vs. control at 24 hours; @elasticity, conventional units: treated vs control at I hour; @@elasticity, conventional units: treated vs control at 24 hours. 
in the diaper environment or in repeated skin washing, for example, in newborn infants. The National Institute for Clinical Excellence guideline on postnatal care ${ }^{24}$ states that a cleaning agent should not be added to a baby's bath water unless it is a mild non-perfumed soap. ${ }^{25}$ However, water alone is insufficient for the removal of oil-soluble skin surface impurities. ${ }^{26-28}$ Depending on bathing frequency and water quality, washing with water alone can lead to skin dryness, ${ }^{29}$ which may adversely affect infant skin condition. In addition, many soaps are alkaline $(\mathrm{pH}>7)$, potentially able to disrupt skin surface $\mathrm{pH}$, alter skin lipids, and cause dryness and irritation. ${ }^{30}$ In this scenario, our results may provide a set of quantitative biophysical parameters to define the state of healthy neonatal skin during transition, when skin maturation is influenced by the water content of the stratum corneum. ${ }^{30}$

In this study, the increase in skin hydration on the heel site was proportionate to age and was physiologic between treated heels and the control ones. However, different from previous studies (all evaluating at birth skin $\mathrm{pH}$ and reporting near-neutral value [pH 6.2-7.5] and reaching adults' level [ $\mathrm{pH}$ 5.4-5.9] after a few weeks), ${ }^{31}$ our data show that heel skin $\mathrm{pH}$ is lower and increases with increasing postnatal age, indicating excellent post emu oil treatment epidermal barrier integrity at transition from intrauterine to extrauterine life.

Our study had several limitations. It was neither a double-blind, randomized controlled trial nor a trial that assessed product efficacy in different body skin surfaces, regardless that different nurses observed the baseline for any given child as at end line. The number of participants was small. However, in the absence of neonatal skin barrier values, we did not complete a power analysis to determine if the number of participants was sufficient or did not satisfy the accuracy of data analysis. Additionally, a lack of information regarding the frequency and timing of maternal assessments around the infants' bathing routine was a limitation. However, the naturalistic setting of the study made it likely comparable to real-life patterns of infant care and product use. Despite these potential limitations, the inclusion criteria were broad, allowing a representative sampling of healthy term infants, each forming both the case and the control population.

\section{Conclusion}

A skin care moisturizer developed specifically for infants (emu oil-based lotion) seems to be well tolerated during transition from intrauterine to extrauterine life, significantly improving stratum corneum hydration, $\mathrm{pH}$, and elasticity of newborns' skin after 1 and even 24 hours of application. These results provide a set of quantitative biophysical and perceptual parameters to define both the state and the improvement of healthy neonatal moisturized skin barrier integrity. This was previously observed through perceptual visual and tactile observations by independent mothers.

\section{Disclosure}

The authors report no conflicts of interest in this work.

\section{References}

1. Visscher MO, Chatterjee R, Ebel JP, LaRuffa AA, Hoath SB. Biomedical assessment and instrumental evaluation of healthy infant skin. Pediatr Dermatol. 2002;19(6):473-481.

2. Stamatas GN, Nikolovski J, Mack MC, Kollias N. Infant skin physiology and development during the first years of life: a review of recent findings based on in vivo studies. Int J Cosmet Sci. 2011;33(1):17-24.

3. Stamatas GN, Nikolovski J, Luedtke MA, Kollias N, Wiegand BC. Infant skin microstructure assessed in vivo differs from adult skin in organization and at the cellular level. Pediatr Dermatol. 2010;27(2):125-131.

4. Fluhr JW, Darlenski R, Taieb A, et al. Functional skin adaptation in infancy - almost complete but not fully competent. Exp Dermatol. 2010;19(6):483-492.

5. Visscher MO, Adam R, Brink S, Odio M. Newborn infant skin: physiology, development, and care. Clin Dermatol. 2015;33(3):271-280.

6. Darmstadt GL, Saha SK, Ahmed AS, et al. Effect of topical treatment with skin barrier-enhancing emollients on nosocomial infections in preterm infants in Bangladesh: a randomised controlled trial. Lancet. 2005;365(9464):1039-1045.

7. Garcia Bartels N, Scheufele R, Prosch F, et al. Effect of standardized skin care regimens on neonatal skin barrier function in different body areas. Pediatr Dermatol. 2010;27(1):1-8.

8. Yonezawa K, Haruna M, Shiraishi M, Matsuzaki M, Sanada H. Relationship between skin barrier function in early neonates and diaper dermatitis during the first month of life: a prospective observational study. Pediatr Dermatol. 2014;31(6):692-697.

9. McManus Kuller J. Update on newborn bathing. Newborn Infant Nurs Rev. 2014;14(4):166-170.

10. Alonso C, Larburu I, Bon E, et al. Efficacy of petrolatum jelly for the prevention of diaper rash: a randomized clinical trial. J Spe Pediatr Nurs. 2013;18(2):123-132.

11. Larson AA, Dinulos JG. Cutaneous bacterial infections in the newborn. Curr Opin Pediatr. 2005;17(4):481-485.

12. Sales J. The emu (Dromaius novaehollandiae): a review of its biology and commercial products. Br Poult Sci. 2007;18:1-20.

13. Jeengar MK, Kumar PS, Thummuri D, et al. Review on emu products for use as complementary and alternative medicine. Nutrition. 2014;31(1):21-27.

14. Bennett DC, Code WE, Godin DV, Cheng KM. Comparison of the antioxidant properties of emu oil with other avian oils. Anim Prod Sci. 2008;48:1345-1350.

15. Zemtsov A, Gaddis M, Montalvo-Lugo V. Moisturizing and cosmetic properties of emu oil: a pilot double blind study. Australas J Dermatol. 1996;37(3):159-161.

16. Fiocchidiriso [webpage on the Internet]. ELLEDIFRI Laboratories. Italy: 2017. Available from: http://www.fiocchidiriso.com/prodotti/ olio-emudermico. Accessed July 11, 2017.

17. callegari1930 [homepage on the Internet]. Soft Plus 5.5 Callegari. Parma, Italy: SPA; 2017. Available from: http://www.callegari1930. com. Accessed July 11, 2017.

18. Snowden JM, Whitehouse MW. Anti-inflammatory activity of emu oils in rats. Inflammopharmacology. 1997;5(2):127-132. 
19. Attarzadeh Y, Asilian A, Shahmoradi Z, Adibi N. Comparing the efficacy of emu oil with clotrimazole and hydrocortisone in the treatment of seborrheic dermatitis: a clinical trial. JRes Med Sci. 2013;18(6):477-481.

20. Whitehouse MW, Turner AG, Davis CK, Roberts MS. Emu oil(s): a source of non-toxic transdermal anti-inflammatory agents in aboriginal medicine. Inflammopharmacology. 1998;6(1):1-8.

21. Knor T, Meholjić-Fetahović A, Mehmedagić A. Stratum corneum hydration and skin surface $\mathrm{pH}$ in patients with atopic dermatitis. Acta Dermatovenerol Croat. 2011;19(4):242-247.

22. Kim DW, Park JY, Na GY, Lee SJ, Lee WJ. Correlation of clinical features and skin barrier function in adolescent and adult patients with atopic dermatitis. Int J Dermatol. 2006;45(5):698-701.

23. Zanardo V, Giarrizzo D, Maiolo L, Straface G. Efficacy of topical application of emu oil on areola skin barrier in breastfeeding women J Evid Based Complementary Altern Med. 2016;21(1):10-13.

24. National Institute for Clinical Excellence. Guideline Development Methods: Information for National Collaborating Centres and Guideline Developers. 2006. Available from: https://www.nice.org.uk/media/ default/about/what-we-do/our-programmes/developing-nice-guidelinesthe-manual.pdf. Accessed July 11, 2017.
25. Iarkowsky LE, Tierney NK, Horowitz P. Tolerance of skin care regimen in healthy, full-term neonates. Clin Cosmet Investig Dermatol. 2013;6:137-144.

26. Gelmetti C. Skin cleansing in children. J Eur Acad Dermatol Venereol. 2001;15(1):12-15.

27. Kuehl BL, Fyfe KS, Shear NH. Cutaneous cleansers. Skin Therapy Lett. 2003;8(3):1-4.

28. Afsar FS. Skin care for preterm and term neonates. Clin Exp Dermatol. 2009;34(8):855-858.

29. Blume-Peytavi U, Cork MJ, Faergemann J, Szczapa J, Vanaclocha F, Gelmetti C. Bathing and cleansing in newborns from day 1 to first year of life: recommendations from a European round table meeting. J Eur Acad Dermatol Venereol. 2009;23(7):751-759.

30. Darmstadt GL, Dinulos JG. Neonatal skin care. Pediatr Clin North Am. 2000;47(4):757-782.

31. Ludriksone L, Garcia Bartels N, Kanti V, Blume-Peytavi U, Kottner J. Skin barrier function in infancy: a systematic review. Arch Dermatol Res. 2014;306(7):591-599.
Clinical, Cosmetic and Investigational Dermatology

\section{Publish your work in this journal}

Clinical, Cosmetic and Investigational Dermatology is an international, peer-reviewed, open access, online journal that focuses on the latest clinical and experimental research in all aspects of skin disease and cosmetic interventions. This journal is included on PubMed. The manuscript management system is completely online

\section{Dovepress}

and includes a very quick and fair peer-review system, which is all easy to use. Visit http://www.dovepress.com/testimonials.php to read real quotes from published authors 